\title{
Space Charge Impedance and Transverse Beam Instability
}

\author{
V.Balbekov \\ Fermi National Accelerator Laboratory \\ P.O. Box 500, Batavia, Illinois 60510
}

February 24, 2006

\begin{abstract}
The role of space charge field in transverse beam instability is revised including beam coupling impedance calculation and Landau damping analysis.
\end{abstract}

\section{Introduction}

Transverse space charge impedance is traditionally treated interchangeably with any other beam coupling impedance, which statement is reflected in handbook [1]. In fact it is rather peculiar, at least the space charge field is evidently non-dipole, i.e. it essentially depends on coordinates in the beam area - in contrast with resistive wall field, for example. Numerically the coherent space charge tune shift is identified with incoherent tune shift (with opposite sign). Because the last typically depends on amplitude, the shift of small incoherent oscillations is accepted without a strong substantiation. Nonlinearity of the space charge field and its possible effect on Landau damping is another problem. Both of them are especially important because space charge impedance dominates very frequently in a budget of impedances. 


\section{Particle and beam betatron oscillations}

In a smooth approximation, betatron oscillations of a particle are described by the equation:

$$
\frac{d^{2} x}{d t^{2}}+\Omega^{2}(p) Q^{2}(p) x=\frac{e E\left(x-x_{c}\right)}{m \gamma^{3}}+A x_{c}
$$

where $\Omega(p)$ and $Q(p)$ are momentum dependent angular velocity and betatron tune of the particle, $x_{c}$ is position of the beam center, and $A x_{c}$ is a contribution of other than space charge sources, first of all walls of the vacuum chamber including resistivity. It is assumed that these sources create dipole field which is proportional to $x_{c}$ and does not depend on $x$. However, a similar assumption is not acceptable to the space charge field $E\left(x-x_{c}\right)$ which is a nonlinear function, with the exception of uniform elliptical beam.

Let us consider all particles with longitudinal momentum $p$ denoting corresponding normalized transverse distribution functions as $F_{\perp}\left(x-x_{p}\right)$ where $x_{p}$ is center of this group of particles, i.e. ${ }^{1}$

$$
x_{p}=\int F_{\perp}\left(x-x_{p}\right) x d x
$$

(actual variables are shown only). Then averaging of Eq. (1) gives:

$$
\frac{d^{2} x_{p}}{d t^{2}}+\Omega^{2}(p) Q^{2}(p) x_{p}=\frac{e}{m \gamma^{3}} \int F_{\perp}(x) E\left(x+x_{p}-x_{c}\right) d x+A x_{c}
$$

Assuming both $x_{p}$ and $x_{c}$ are small, expand function $E$ in Taylor series. Then, taking into account that $F_{\perp}(x)$ and $E(x)$ are even and odd functions, one can bring Eq. (3) to the form:

$$
\frac{d^{2} x_{p}}{d t^{2}}+\Omega^{2}(p) Q^{2}(p) x_{p}=2 \Omega_{c}^{2} Q_{c} \Delta Q\left[x_{p}-x_{c}\right]+A x_{c}
$$

where $\Omega_{c}$ and $Q_{c}$ are central values and

$$
2 \Omega_{c}^{2} Q_{c} \Delta Q=\frac{e}{m \gamma^{3}} \int F_{\perp}(x) E^{\prime}(x) d x
$$

\footnotetext{
${ }^{1}$ We assume that this function does not depend on $p$ explicitly because similar dependence would mean transverse-longitudinal correlation which is rather special case.
} 
Next we take into account that

$$
\frac{d}{d t}=\frac{\partial}{\partial t}+\Omega(p) \frac{\partial}{\partial \theta}
$$

where $\theta$ is azimuth, and consider solution $\propto \exp (i k \theta-i \omega t)$. Relation of amplitudes following from Eq. (4) can be written in the form:

$$
x_{p}=\frac{\left[2 \Omega_{c}^{2} Q_{c} \Delta Q-A\right] x_{c}}{[\omega-k \Omega(p)]^{2}-\Omega^{2}(p)[Q(p)-\Delta Q]^{2}}
$$

Multiplying it on normalized distribution function of momentum $F_{\|}(p)$ and integrating, one obtains dispersion equation of coherent oscillations

$$
1=\left[2 \Omega_{c}^{2} Q_{c} \Delta Q-A\right] \int_{-\infty}^{\infty} \frac{F_{\|}(p) d p}{[\omega-k \Omega(p)]^{2}-\Omega^{2}(p)[Q(p)-\Delta Q]^{2}}
$$

where the relation

$$
x_{c}=\int_{-\infty}^{\infty} F_{\|}(p) x_{p} d p
$$

is taken into account. With a reasonable accuracy, Eq. (8) can be represented in more traditional form:

$$
1=\mp\left[\Omega_{c} \Delta Q-\frac{A}{2 \Omega_{c} Q_{c}}\right] \int_{-\infty}^{\infty} \frac{F_{\|}(p) d p}{\omega-\Omega(p)[k \mp[Q(p)-\Delta Q]]}
$$

\section{Beam coupling impedance}

Comparison of Eq. (10) with known dispersion equation of transverse instability allows us to write following expression for space charge impedance:

$$
Z^{(s c)}=\frac{i \gamma Z_{0} Q_{c} \Delta Q}{r_{0} R_{0} N^{\prime}}
$$

where $Z_{0}=4 \pi / c \simeq 377 \mathrm{Ohm}, r_{0}=e^{2} / m c^{2}\left(1.535 \times 10^{-16} \mathrm{~cm}\right.$ for protons $)$, $R_{0}$ is average radius, and $N^{\prime}$ is linear density of particles. 
Further we need to use some beam model to calculate the beam field $E$ and tune shift $\Delta Q$. For a round beam with normalized space charge density $\rho(r)$, horizontal field strength is:

$$
E=\frac{4 \pi e N^{\prime} x}{r^{2}} \int_{0}^{r} \rho\left(r^{\prime}\right) r^{\prime} d r^{\prime}
$$

Therefore, using Eq. (5) one can represent the tune shift in the form:

$$
\Delta Q=\frac{2 \pi^{2} r_{0} R_{0}^{2} N^{\prime}}{\beta^{2} \gamma^{3} Q_{c}} \int_{0}^{\infty} \rho^{2}(r) r d r
$$

Two cases are considered below:

$\underline{\text { Uniform beam of radius a }}$

$$
\rho(r)=\frac{1}{\pi a^{2}} \quad \text { at } \quad r<a
$$

Then

$$
\Delta Q=\frac{r_{0} R_{0}^{2} N^{\prime}}{\beta^{2} \gamma^{3} a^{2} Q_{c}} \quad \text { and } \quad Z^{(s c)}=\frac{i Z_{0} R_{0}}{\beta^{2} \gamma^{2} a^{2}}
$$

First of these expressions is incoherent tune shift (with opposite sign), which does not depend on amplitude in this case. Second formula coincides with usually used one [1].

Gaussian beam

$$
\rho(r)=\frac{1}{2 \pi \sigma^{2}} \exp \left(-\frac{r^{2}}{2 \sigma^{2}}\right)
$$

Then

$$
\Delta Q=\frac{r_{0} R_{0}^{2} N^{\prime}}{4 \beta^{2} \gamma^{3} \sigma^{2} Q_{c}} \quad \text { and } \quad Z^{(s c)}=\frac{i Z_{0} R_{0}}{4 \beta^{2} \gamma^{2} \sigma^{2}}
$$

First of these expressions is exactly 2 times less of incoherent tune shift of small oscillations, and beam coupling impedance is correspondingly twice less of cited in Ref. [1]. 


\section{About Landau damping}

Incoherent tune shift depends on amplitude of betatron oscillations excluding unrealistic case of elliptical beam with constant density. However, it does not manifest in dispersion equation (10) though the nonlinearity of the field $E$ is presumed in basic Eq. (1). It means that this nonlinearity does not contribute to coherent effects and has not to be included to calculation of a tune spread at the analysis of Landau damping. The reason is that this nonlinearity - in contrast with external one - moves with the beam and cannot have an effect on this coherent motion.

\section{References}

[1] "Handbook of Accelerator Physics and Engineering", Edited by A. Chao and M. Tigner, World Scientific 1998, p. 112. 